\title{
Observer-based Variable Universe Adaptive Fuzzy Controller Without Additional Dynamic Order
}

\author{
Hai-Gang Guo ${ }^{1}$ Bao-Jie Zhang ${ }^{2}$ \\ ${ }^{1}$ School of Mathematics and Statistics, Henan University of Science and Technology, Luoyang 471023, China \\ ${ }^{2}$ School of Mathematics and Information Science, Qujing Normal University, Qujing 655011, China
}

\begin{abstract}
A high-precision fuzzy controller, based on a state observer, is developed for a class of nonlinear single-input-single-output (SISO) systems with system uncertainties and external disturbances. The state observer is introduced to resolve the problem of the unavailability of state variables. Assisted by the observer, a variable universe fuzzy system is designed to approximate the ideal control law. Being auxiliary components, a robust control term and a state feedback control term are designed to suppress the influence of the lumped uncertainties and remove the observation error, respectively. Different from the existing results, no additional dynamic order is required for the control design. All the adaptive laws and the control law are built based on the Lyapunov synthesis approach, and the signals involved in the closed-loop system are guaranteed to be uniformly ultimately bounded. Simulation results performed on Duffing forced oscillation demonstrate the advantages of the proposed control scheme.
\end{abstract}

Keywords: Variable universe fuzzy system, observer, strictly positive real (SPR) condition, dynamic order, $\sigma$-modification, adaptive bounding technique.

\section{Introduction}

In recent decades, fuzzy control has been a hot research topic in the nonlinear control field. The main reason lies in the facts that fuzzy systems own the universal approximation property and they can convert the human linguistic concepts into automatic control strategies ${ }^{[1]}$. A great deal of work has demonstrated their advantage and feasibility for modeling and control of uncertain complex nonlinear systems ${ }^{[2-7]}$. However, everything has two sides. A fuzzy controller is essentially an interpolator ${ }^{[8]}$. The fuzzy controller derived from human experts, which has a limited number of control rules, is subject to lower controlling precision $^{[8,9]}$. This disadvantage often makes fuzzy control difficult to meet the need of engineering practice ${ }^{[7,10]}$. Subsequently, several approaches are proposed to improve the control precision of fuzzy control such as PID-based fuzzy control schemes ${ }^{[11]}$, optimizing membership function parameters $^{[12,13]}$, small error interpolation ${ }^{[14]}$, etc.

Among various high-precision fuzzy control schemes, the variable universe adaptive fuzzy control (VUAFC) works in the most possible intuitive manner. According to the interpolation mechanism ${ }^{[8]}$, achieving high controlling precision inevitably requires a large number of fuzzy rules. Yet the simple increase in the number of fuzzy rules by adding the domain division points may cause the so-called "dimension curse" problem. In fact, control is essentially a local problem since the control purpose is generally to ensure the tracking error asymptotically converging to a neighborhood of zero. To achieve high-precision control, we only need to ensure enough fuzzy rules around zero. Based on this methodology, $\mathrm{Li}^{[15]}$ first presented an adaptive fuzzy

Regular paper

Manuscript received January 15, 2013; revised November 15, 2013

This work was supported by National Natural Science Foundation of China (No.61074044), and Basic and Cutting-edge Technology of Science and Technology Department of Henan Province (No. 092300410178). control scheme with variable universe. The idea behind the scheme is that the universe of discourse adaptively contracts with the error decreasing. Under the same rule number, the universe contraction means that the number of fuzzy rules around zero increases. Consequently, VUAFC relatively increases the number of fuzzy rules and improves the control precision. At present, VUAFC has been widely used in the inverted pendulum ${ }^{[16,17]}$, aerospace vehicle ${ }^{[18]}$, chaotic system $^{[19-21]}$, near space vehicle ${ }^{[22]}$, etc.

A common assumption for these schemes ${ }^{[16-22]}$ is that the state vector is assumed to be available for measurement. Unfortunately, in engineering practice, due to size and cost, the physical state variables may be partially or fully unavailable for measurement. The problem was solved in [23-26] by introducing a state or tracking error observer in their adaptive fuzzy control schemes. To use the Meyer-KalmomYacubovich (MKY) lemma, in these schemes, the strictly positive real (SPR) condition on the observation error dynamics must be satisfied. The need for SPR condition results in the filtering of the fuzzy basis function (FBF), which makes the dynamic order of the observer-controller very large ${ }^{[27]}$. In addition, as stated in $[28,29]$, these observerbased fuzzy adaptive controllers have not been derived rigorously in mathematics. Aiming at these disadvantages, the authors in $[27,29,30]$ employed the state variable filters or the tracking error filter to design the update law and the robust control term. In their solutions, no SPR condition is needed and the filtering of FBF can be effectively avoided. However, to some degree, additional dynamic order is still required in their design schemes. The need increases the design difficulty of the controller-observer. In the present study, we try to solve these disadvantages based on the variable universe adaptive fuzzy output feedback control. To the best of our knowledge, there are a few results on variable universe adaptive fuzzy control for nonlinear system with immeasurable states. In [31], an observer-based robust variable universe adaptive fuzzy controller was de- 
veloped for a chaotic system. However, due to the absence of the SPR condition, the parameter adaptive laws and the control laws in the control design cannot be implemented.

In this paper, a novel fuzzy output feedback controller is developed for a class of single input single output (SISO) nonlinear systems with function uncertainties and external disturbances. A state observer is designed to estimate the tracking error. Based on the observer, a variable universe adaptive fuzzy system is used to approximate the unknown ideal control law. Contrast to the work in [23-26], two amendments are made to deal with the two disadvantages above. One amendment is to amend the ideal control law to the form of (7). This amendment, which is described in Remark 2, makes the design of the observer-controller more vigorous in mathematics. The other is that a robust adaptive control term is augmented to eliminate the effect of the lump uncertainties including the filtering of FBF and other functions. Consequently, there is no increase in the dynamic order of the observer-controller. Based on the SPR-Lyapunov design approach, the proposed controller can guarantee that tracking error converges to the small neighborhood of zero and the involved signals are all uniformly ultimately bounded. Simulation results also demonstrate the superior properties of the proposed scheme over the counterpart of fuzzy control.

The remainder of the paper is organized as follows: in Sections 2 and 3, brief statements about the control system and variable universe adaptive fuzzy system are provided, respectively. We develop an observer-based variable universe adaptive fuzzy controller for a class of SISO nonlinear systems with unmeasurable states in Section 4. Simulation results for the proposed controller are illustrated in Section 5. Section 6 concludes this paper.

\section{Problem formulation}

In this paper, we consider the nonlinear system as follows:

$$
\begin{aligned}
& x^{(n)}=f\left(x, \dot{x}, \cdots, x^{(n-1)}\right)+b u+d \\
& y=x .
\end{aligned}
$$

Or equivalently, system (1) can be rewritten into the following form:

$$
\begin{aligned}
& \dot{\boldsymbol{x}}=A \boldsymbol{x}+B[f(\boldsymbol{x})+b u+d] \\
& y=C^{\mathrm{T}} \boldsymbol{x}
\end{aligned}
$$

where

$A=\left[\begin{array}{ccccc}0 & 1 & 0 & \cdots & 0 \\ 0 & 0 & 1 & \cdots & 0 \\ \vdots & \vdots & \vdots & \ddots & \vdots \\ 0 & 0 & 0 & \cdots & 1 \\ 0 & 0 & 0 & \cdots & 0\end{array}\right], \quad B=\left[\begin{array}{c}0 \\ 0 \\ \vdots \\ 0 \\ 1\end{array}\right], \quad C=\left[\begin{array}{c}1 \\ 0 \\ \vdots \\ 0 \\ 0\end{array}\right]$.

$\boldsymbol{x}=\left(x_{1}, x_{2}, \cdots, x_{n}\right)^{\mathrm{T}} \triangleq\left(x, \dot{x}, \cdots, x^{(n-1)}\right)^{\mathrm{T}}$ is the state vector of the system which is not available for measurement, and $u \in \mathbf{R}$ and $y \in \mathbf{R}$ are the input and the output of the system, respectively. $f(\boldsymbol{x})$ is an unknown continuous function with upper bound $\bar{f}$ and $b$ is an unknown constant with lower bound $b_{L}$, that is, $|f(\boldsymbol{x})| \leqslant \bar{f}$ and $b \geqslant b_{L}>0 . d(t)$ is a bounded external disturbance and $|d(t)| \leqslant D . \boldsymbol{y}_{r}=\left(y_{r}, \dot{y}_{r}, \cdots, y_{r}^{(n-1)}\right)^{\mathrm{T}}$ is a desired trajectory vector which is supposed to be continuous, bounded and available for measurement. The tracking error is defined as $\boldsymbol{e}=\boldsymbol{y}_{r}-\boldsymbol{x}=\left(e_{1}, e_{2}, \cdots, e_{n}\right)^{\mathrm{T}}$. It is easy to verify that the pair $(A, B)$ is controllable and the pair $\left(C^{\mathrm{T}}, A\right)$ is observable.

The control task is to design a control law such that the system output $y$ follows a specified desired trajectory $y_{r}$ and the signals involved in the closed-loop system remain uniformly ultimately bounded.

\section{Variable universe adaptive fuzzy sys- tem}

The variable universe idea was firstly developed in [15]. Further, in [32] several kinds of variable universe adaptive fuzzy control methods were investigated in detail, which has laid a theoretical foundation for the application of VUAFC. Facts have proved that VUAFC can be well applied to control of a wide class of uncertain nonlinear systems. In particular, it has shown several excellent control qualities such as fast dynamic response, high controlling-precision, almost no overshoot, etc. ${ }^{[16,18,20,33,34]}$ The basic structure of variable universe adaptive fuzzy control is briefly introduced in this section.

Let $X_{i}=\left[-E_{i}, E_{i}\right]$ be the universe of input variable $z_{i}(i=1,2, \cdots, m)$ and $Y=[-U, U]$ the universe of output variable $u_{o} . \mathcal{A}_{i}=\left\{A_{i j}\right\}(j=1,2, \cdots, N)$ is defined as a fuzzy partition on $X_{i}$ and $\mathcal{B}=\left\{B_{j}\right\}(j=1,2, \cdots, N)$ a fuzzy partition on $Y$, where $A_{i j} \in \mathcal{F}\left(X_{i}\right)$ and $B_{j} \in \mathcal{F}(Y)$ are termed as the base, and $a_{i j}$ and $b_{j}$ are the peak points of $A_{i j}$ and $B_{j}$, respectively. $\mathcal{A}_{i}(i=1,2, \cdots, m)$ and $\mathcal{B}$ are regarded as linguistic variables, so that a group of fuzzy inference rules are formed as follows:

$$
\begin{aligned}
& \text { If } z_{i} \text { is } A_{1 j}, z_{2} \text { is } A_{2 j}, \cdots, \text { and } z_{m} \text { is } A_{m j}, \\
& \text { then } u_{o} \text { is } B_{j}(j=1,2, \cdots, N)
\end{aligned}
$$

where $N$ represents the number of the rules. Singleton fuzzifier, triangle membership function (overlap law is 0.5), product inference engine, and center average defuzzifier are used in this fuzzy system. The derived output of the fuzzy system is

$$
\hat{u}_{o}(z)=\xi(z)^{\mathrm{T}} \theta
$$

where $\theta=\left[b_{1}, b_{2}, \cdots, b_{N}\right]^{\mathrm{T}}$ denotes the parameter vector and $\xi(z)=\left[\xi_{1}(z), \xi_{2}(z), \cdots, \xi_{N}(z)\right]^{\mathrm{T}}$ denotes the fuzzy base function vector with $\xi_{j}(z)=\prod_{i=1}^{m} A_{i j}\left(z_{i}\right)(j=1,2, \cdots, N)$.

The so-called variable universe means that some universes, for example $X_{i}$ and $Y$, respectively, can change along with changing of variables $z_{i}$ and $u_{o}$. In this case, the variable universes are denoted by

$$
X_{i}\left(z_{i}\right)=\left[-\alpha_{i}\left(z_{i}\right) E_{i}, \alpha_{i}\left(z_{i}\right) E_{i}\right]
$$

and

$$
Y\left(u_{o}\right)=\left[-\beta\left(u_{o}\right) U, \beta\left(u_{o}\right) U\right]
$$

where $\alpha_{i}\left(z_{i}\right)$ and $\beta\left(u_{o}\right)$ are, respectively, called contractionexpansion factors of the universes $X_{i}$ and $Y$. Being relative to the variable universes, the original universes $X_{i}$ and $Y$ are naturally called initial universes. After the above 
changes, the output of variable universe fuzzy system becomes

$$
\hat{u}_{o}(z \mid \beta)=\beta \zeta(z)^{\mathrm{T}} \theta
$$

where $\zeta(z)=\left[\zeta_{1}(z), \zeta_{2}(z), \cdots, \zeta_{N}(z)\right]^{\mathrm{T}}$ denotes the fuzzy base function vector with $\zeta_{j}(z)=\prod_{i=1}^{m} A_{i j}\left(\frac{z_{i}}{\alpha_{i}\left(z_{i}\right)}\right)(j=$ $1,2, \cdots, N)$.

Remark 1. Only one parameter, rather than a parameter vector, is needed to be updated online in VUAFC. Therefore, VUAFC scheme reduces the computational burden of adaptive fuzzy control. On the other hand, the design of variable universe fuzzy controller hardly needs smart expert knowledge in the realm, but the rough tendency of rules only ${ }^{[15]}$. From this point, it reduces the design difficulty.

\section{Observer-based variable universe adaptive fuzzy control design}

Based on the feedback linearization approach, when the function $f(\boldsymbol{x})$ and the control gain $b$ in (1) are known, and assuming that $d=0$, the so-called ideal controller can be easily designed as follows:

$$
u^{*}=\frac{1}{b}\left(-f(\boldsymbol{x})+y_{r}^{(n)}+K_{c}^{\mathrm{T}} \boldsymbol{e}\right)
$$

where the feedback gain vector $K_{c}=\left[k_{c, 1}, k_{c, 2}, \cdots, k_{c, n}\right]^{\mathrm{T}}$ is chosen such that the characteristic polynomial $A-B K_{c}^{\mathrm{T}}$ is Hurwitz because $(A, B)$ is controllable.

Substituting (5) into system (1) and using $e=y_{r}-x$ yields

$$
e^{(n)}+K_{c}^{\mathrm{T}} \boldsymbol{e}=e^{(n)}+k_{c, n} e^{(n-1)}+\cdots+k_{c, 1} e=0 .
$$

Thus, the control objective can be achieved. However, since the function $f(\boldsymbol{x})$ and constant $b$ are all unknown and the state vector $\boldsymbol{x}$ is unmeasured, the ideal control law $u^{*}$ can not be implemented. In this situation, we must design a state observer and a control law to solve this problem.

Since the state vector $x$ is unavailable for measurement, motivated by [35], we modify the ideal control law $u^{*}$ in (5) as follows:

$$
u^{*}=\frac{1}{b}\left(-f(\hat{\boldsymbol{x}})+y_{r}^{(n)}+K_{c}^{\mathrm{T}} \hat{\boldsymbol{e}}\right) .
$$

And we design the control law as follows:

$$
u=\hat{u}_{c}(\hat{\boldsymbol{e}} \mid \beta)+u_{r}+u_{a}
$$

where $\hat{u}_{c}(\hat{\boldsymbol{e}} \mid \beta)$ is a variable universe adaptive fuzzy system, as shown in (4), which is employed to approximate the modified control law $u^{*}$ in (7). $u_{r}$ denotes a compensation term to remove the effect of fuzzy approximation error (FAE) and the external disturbances. $u_{a}$ expresses the observer error feedback control term. They will be designed below.

Remark 2. As stated in [28], the ideal control in [23, 26]

$$
u^{*}=\frac{1}{b}\left(-f(\boldsymbol{x})+y_{r}^{(n)}+K_{c}^{\mathrm{T}} \hat{\boldsymbol{e}}\right) .
$$

is questionable. In fact, if substituting (9) into the system equation (1), we find the following dynamics $e^{(n)}+K_{c}^{\mathrm{T}} \hat{\boldsymbol{e}}=0$.
From these dynamics, the conclusion that the convergence of the tracking error to zero does not hold true.

However, by using the modified ideal control law (7), we can obtain the corresponding tracking-error equation

$$
\begin{aligned}
e^{(n)}+K_{c}^{\mathrm{T}} \boldsymbol{e}= & \left(K_{c}^{\mathrm{T}} \boldsymbol{e}-K_{c}^{\mathrm{T}} \hat{\boldsymbol{e}}\right)+(f(\hat{\boldsymbol{x}})-f(\boldsymbol{x}))+ \\
& b\left(u^{*}-\hat{u}_{c}(\hat{\boldsymbol{e}} \mid \beta)\right)-b u_{r}-b u_{a} .
\end{aligned}
$$

From the above equation, we can see that the value of $e^{(n)}+K_{c}^{\mathrm{T}} \hat{\boldsymbol{e}}$ is subjected to the effect of the observation error and FAE. The most common solution is that a compensation term $u_{r}$ is augmented to eliminate these uncertainties. Obviously, if there is no observation error or FAE between $\hat{u}_{c}$ and $u^{*}$, then $e^{(n)}+K_{c}^{\mathrm{T}} \boldsymbol{e}=0$ and in turn $\lim _{t \rightarrow \infty} e=0$ can be derived.

According to the universal approximation theorem ${ }^{[1]}$, the ideal control law $u^{*}$ in (7) can be approximated as follows:

$$
u^{*}=\hat{u}_{c}\left(\hat{\boldsymbol{e}} \mid \beta^{*}\right)+\epsilon
$$

where $\epsilon$ denotes FAE and $\beta^{*}=\arg \min _{\beta \in \Omega_{\beta}}\left\{\sup _{\hat{\boldsymbol{x}} \in \Omega_{\hat{\boldsymbol{x}}}} \mid \hat{u}_{c}(\hat{\boldsymbol{e}} \mid \beta)-\right.$ $\left.u^{*} \mid\right\}$ denotes the optimal contraction-expansion factor with $\Omega_{\hat{\boldsymbol{x}}}$ and $\Omega_{\beta}$ expressing the constraint set of state vector $\hat{\boldsymbol{x}}$ and parameter $\beta$, respectively.

Assumption 1. FAE $\epsilon$ is bounded by an unknown constant $c_{1}$.

Assumption 2. The optimal contraction-expansion factor $\beta^{*}$ is bounded by an unknown constant $M_{\beta}$.

Substituting (7) into (1) and doing some straightforward manipulation, we obtain the tracking error equation

$$
\begin{gathered}
e^{(n)}=-K_{c}^{\mathrm{T}} \hat{\boldsymbol{e}}+(f(\hat{\boldsymbol{x}})-f(\boldsymbol{x}))-d+ \\
b\left(\tilde{\beta} \zeta(\hat{\boldsymbol{e}}, \alpha)^{\mathrm{T}} \theta+\epsilon-u_{r}-u_{a}\right)
\end{gathered}
$$

or equivalently

$$
\begin{aligned}
& \dot{\boldsymbol{e}}=A \boldsymbol{e}-B K_{c}^{\mathrm{T}} \hat{\boldsymbol{e}}+B\left[b\left(\tilde{\beta} \zeta(\hat{\boldsymbol{e}}, \alpha)^{\mathrm{T}} \theta+\epsilon_{1}-u_{r}-u_{a}\right)\right] \\
& e_{1}=C^{\mathrm{T}} \boldsymbol{e}
\end{aligned}
$$

where $\tilde{\beta} \triangleq \beta^{*}-\beta$ and $\epsilon_{1}=\epsilon+\frac{1}{b}(f(\hat{\boldsymbol{x}})-f(\boldsymbol{x})-d)$.

Design the observer as follows:

$$
\begin{aligned}
& \dot{\hat{\boldsymbol{e}}}=A \hat{\boldsymbol{e}}-B K_{c}^{\mathrm{T}} \hat{\boldsymbol{e}}+K_{o}\left(e_{1}-\hat{e}_{1}\right) \\
& \hat{e}_{1}=C^{\mathrm{T}} \hat{\boldsymbol{e}}
\end{aligned}
$$

where $K_{o}=\left[\begin{array}{llll}k_{o, 1} & k_{o, 2} & \cdots & k_{o, n}\end{array}\right]^{\mathrm{T}}$ is the observer gain vector to guarantee the characteristic polynomial of $\left(A-K_{o} C^{\mathrm{T}}\right)$ to be Hurwitz because $(C, A)$ is observable. If the observation error is defined as $\tilde{\boldsymbol{e}}=\boldsymbol{e}-\hat{\boldsymbol{e}}$, subtracting (12) from (11) yields

$$
\begin{aligned}
& \dot{\tilde{\boldsymbol{e}}}=\left(A-K_{o} C^{\mathrm{T}}\right) \tilde{\boldsymbol{e}}+B\left[b\left(\tilde{\beta} \zeta(\hat{\boldsymbol{e}}, \alpha)^{\mathrm{T}} \theta+\epsilon_{1}-u_{r}-u_{a}\right)\right] \\
& \tilde{e}_{1}=C^{\mathrm{T}} \tilde{\boldsymbol{e}} .
\end{aligned}
$$

Since only the observation output $\tilde{e}_{1}$ in (13) is measurable, we use the SPR-Lyapunov design approach to analyze the stability of the observation error dynamics (13) and generate the adaptive law for parameter $\beta$.

The observation error dynamics (13) can be expressed as

$$
\tilde{e}_{1}=H(s)\left[b\left(\tilde{\beta} \zeta(\hat{\boldsymbol{e}}, \alpha)^{\mathrm{T}} \theta+\epsilon_{1}-u_{r}-u_{a}\right)\right]
$$


where

$$
\begin{aligned}
H(s)= & C^{\mathrm{T}}\left(s I-\left(A-K_{o} C^{\mathrm{T}}\right)\right)^{-1} B= \\
& \frac{1}{s^{n}+k_{o, 1} s^{n-1}+\cdots+k_{o, n}} .
\end{aligned}
$$

In general, the observation error dynamics are not SPR. In order to use SPR-Lyapunov design approach, here we introduce an SPR filter $L(s)$. Equation (14) can be written as

$$
\tilde{e}_{1}=H(s) L(s)\left[b\left(\tilde{\beta} \zeta(\hat{\boldsymbol{e}}, \alpha)^{\mathrm{T}} \theta+\epsilon_{2}-u_{r}-u_{a}\right)\right]
$$

where $\epsilon_{2}=-\tilde{\beta} \zeta(\hat{\boldsymbol{e}}, \alpha)^{\mathrm{T}} \theta+u_{r}+u_{a}+L^{-1}(s)\left[\tilde{\beta} \zeta(\hat{\boldsymbol{e}}, \alpha)^{\mathrm{T}} \theta+\right.$ $\left.\epsilon_{1}-u_{r}-u_{a}\right]$ denotes lumped uncertainties including FAE, external disturbances, and some control terms to be design. Filter $L(s)$ is chosen so that $L^{-1}(s)$ is a proper stable transfer function and $H(s) L(s)$ is a proper SPR transfer function. Let

$$
L(s)=s^{m}+b_{1} s^{m-1}+\cdots+b_{m}, \quad(m=n-1) .
$$

The state-space realization of (14) can be written as

$$
\begin{aligned}
& \dot{\tilde{\boldsymbol{e}}}_{s}=A_{s} \tilde{\boldsymbol{e}}_{s}+B_{s}\left[b\left(\tilde{\beta} \zeta(\hat{\boldsymbol{e}}, \alpha)^{\mathrm{T}} \theta+\epsilon_{2}-u_{r}-u_{a}\right)\right] \\
& \tilde{e}_{1}=C_{s}^{\mathrm{T}} \tilde{\boldsymbol{e}}_{s}
\end{aligned}
$$

where $A_{s}=A-K_{o} C^{\mathrm{T}}, B_{s}=\left[\begin{array}{llll}1 & b_{1} & \cdots & b_{m}\end{array}\right]^{\mathrm{T}}, C_{s}=C$.

Remark 3. According to the work in $[27,28,36]$, in the control design, the filtered signals of FBF vector, $u_{r}$, and $u_{a}$ are all included in the lumped uncertainty $\epsilon_{2}$. The filter $L^{-1}(s)$ appearing in the lumped uncertainty $\epsilon_{2}$ is just for analysis purpose. Thus, the filtering of the FBF vector can be avoided. Therefore, the use of SPR condition in our proposed scheme does not result in an additional increment of the dynamic order of the observer-controller.

Assumption 3. $P_{1}$ and $P_{2}$ are the positive define solutions satisfying the following Riccati equation

$$
\begin{aligned}
& \left(A-B K_{c}^{\mathrm{T}}\right)^{\mathrm{T}} P_{1}+P_{1}\left(A-B K_{c}^{\mathrm{T}}\right)+Q_{1}=0 \\
& \left\{\begin{array}{l}
A_{s}^{\mathrm{T}} P_{2}+P_{2} A_{s}+Q_{2}=0 \\
P_{2} B_{s}=C_{s} .
\end{array}\right.
\end{aligned}
$$

Since the observer output error $\tilde{e}_{1}$ is available, the parameter update law and the state feedback control $u_{a}$ can be designed as follows:

$$
\begin{aligned}
& \dot{\beta}=\gamma_{1}\left(\tilde{e}_{1} \zeta(\hat{\boldsymbol{e}})^{\mathrm{T}} \theta-\sigma\left(\beta-\beta^{0}\right)\right), \\
& u_{a}=K_{o}^{\mathrm{T}} P_{1} \hat{\boldsymbol{e}}
\end{aligned}
$$

where $\gamma_{1}>0, \sigma>0$ and $\beta^{0}$ are design constants.

As indicated in [37], the traditional adaptive laws in the literature may lead to the update parameters unbounded due to the presence of uncertainties in the error equation. The uncertainties include the observation error, FAE, external disturbances, etc. One way to counteract the effect of the uncertainties is to modify the adaptive law using leakage, dead zone, projection, etc. Here, we modify the traditional adaptive law using the fixed $\sigma$-modification. The parameter $\beta^{0}$ denotes an educated guess of $\beta$. It may derive from the experience of human experts.
Assumption 4. The lumped uncertainty $\epsilon_{2}$ is bounded, that is, $\left|\epsilon_{2}\right| \leqslant c_{2}|\tilde{\beta}|+c_{3}$, where $c_{2}$ and $c_{3}$ are unknown positive constants.

The adaptive law with $\sigma$ modification in (19) guarantees the boundedness of parameter $\beta$. Together with Assumption 2, i.e., $\left|\beta^{*}\right| \leqslant M_{\beta}$, then $\tilde{\beta}$ is bounded. Further, combining Assumption 4, we can choose constant $\varepsilon^{*}$ as the optimal upper bound of $\epsilon_{2}$. Due to the unknown parameter $\varepsilon^{*}$, we estimate it with $\varepsilon$. Then the robust control term $u_{r}$ can be designed as

$$
u_{r}=\varepsilon \operatorname{sgn}\left(\tilde{e}_{1}\right)
$$

where the adaptive laws of parameter $\varepsilon$ is designed as follows

$$
\dot{\varepsilon}=\gamma_{2}\left(\left|\tilde{e}_{1}\right|-\sigma\left(\varepsilon-\varepsilon^{0}\right)\right)
$$

where $\gamma_{2}>0$ and $\varepsilon^{0}$ are design constants.

Theorem 1. Consider the nonlinear system (1) with the control law (8), where $\left.\hat{u}_{c}(\hat{\boldsymbol{e}} \mid \beta)\right), u_{r}$, and $u_{a}$ are given as (4), (21), and (20), respectively. The adaptive laws are chosen as (19) and (22). If Assumptions 1-4 hold, then the signals involved in the closed-loop system and the adaptive parameters are all uniformly ultimately bounded.

Proof. Noting that $\tilde{\beta}=\beta^{*}-\beta$ and $\tilde{\varepsilon}=\varepsilon^{*}-\varepsilon$, we choose Lyapunov function as follows:

$$
V=\frac{1}{2} \hat{\boldsymbol{e}}^{\mathrm{T}} P_{1} \hat{\boldsymbol{e}}+\frac{1}{2 b} \tilde{\boldsymbol{e}}_{s}^{\mathrm{T}} P_{2} \tilde{\boldsymbol{e}}_{s}+\frac{1}{2 \gamma_{1}} \tilde{\beta}^{2}+\frac{1}{2 \gamma_{2}} \tilde{\varepsilon}^{2} .
$$

By using (12), (16)-(18), the derivative of $V$ with respect to time yields

$$
\begin{aligned}
\dot{V}= & \frac{1}{2} \dot{\boldsymbol{e}}^{\mathrm{T}} P_{1} \hat{\boldsymbol{e}}+\frac{1}{2} \hat{\boldsymbol{e}}^{\mathrm{T}} P_{1} \dot{\hat{\boldsymbol{e}}}+\frac{1}{2 b} \dot{\tilde{\boldsymbol{e}}}_{s}^{\mathrm{T}} P_{2} \tilde{\boldsymbol{e}}_{s}+ \\
& \frac{1}{2 b} \tilde{\boldsymbol{e}}_{s}^{\mathrm{T}} P_{2} \dot{\tilde{\boldsymbol{e}}}_{s}-\frac{1}{\gamma_{1}} \tilde{\beta} \dot{\beta}-\frac{1}{\gamma_{2}} \tilde{\varepsilon} \dot{\varepsilon}= \\
& \frac{1}{2} \hat{\boldsymbol{e}}^{\mathrm{T}}\left[\left(A-B K_{c}^{\mathrm{T}}\right)^{\mathrm{T}} P_{1}+P_{1}\left(A-B K_{c}^{\mathrm{T}}\right)\right] \hat{\boldsymbol{e}}+ \\
& \hat{\boldsymbol{e}}^{\mathrm{T}} P_{1} K_{o} C^{\mathrm{T}} \tilde{\boldsymbol{e}}_{s}-\frac{1}{\gamma_{1}} \tilde{\boldsymbol{\beta}} \dot{\beta}-\frac{1}{\gamma_{2}} \tilde{\varepsilon} \dot{\varepsilon} \\
& \frac{1}{2 b} \tilde{\boldsymbol{e}}_{s}^{\mathrm{T}}\left[\left(A-K_{o} C^{\mathrm{T}}\right)^{\mathrm{T}} P_{2}+P_{2}\left(A-K_{o} C^{\mathrm{T}}\right)\right] \tilde{\boldsymbol{e}}_{s}+ \\
& \tilde{e}_{1}\left[\epsilon_{2}+\tilde{\beta} \zeta(\hat{\boldsymbol{e}}, \alpha)^{\mathrm{T}} \theta-u_{r}-u_{a}\right]= \\
& -\frac{1}{2} \hat{\boldsymbol{e}}^{\mathrm{T}} Q_{1} \hat{\boldsymbol{e}}-\frac{1}{2 b} \tilde{\boldsymbol{e}}_{s}^{\mathrm{T}} Q_{2} \tilde{\boldsymbol{e}}_{s}+\left(\hat{\boldsymbol{e}}^{\mathrm{T}} P_{1} K_{o} C^{\mathrm{T}} \tilde{\boldsymbol{e}}_{s}-\tilde{e}_{1} u_{a}\right)+ \\
& \frac{1}{\gamma_{1}} \tilde{\beta}\left(\gamma_{1} \tilde{e}_{1} \zeta(\hat{\boldsymbol{e}}, \alpha)^{\mathrm{T}} \theta-\dot{\beta}\right)+\left(\tilde{e}_{1}\left(\varepsilon-u_{r}\right)-\frac{1}{\gamma_{2}} \tilde{\varepsilon} \dot{\varepsilon}\right) .
\end{aligned}
$$

Substituting (19) and (22) into the above equation yields

$$
\begin{aligned}
\dot{V} \leqslant & -\frac{1}{2} \hat{\boldsymbol{e}}^{\mathrm{T}} Q_{1} \hat{\boldsymbol{e}}-\frac{1}{2 b} \tilde{\boldsymbol{e}}_{s}^{\mathrm{T}} Q_{2} \tilde{\boldsymbol{e}}_{s}+\frac{1}{\gamma_{1}} \tilde{\beta}\left(\gamma_{1} \tilde{e}_{1} \zeta(\hat{\boldsymbol{e}}, \alpha)^{\mathrm{T}} \theta-\dot{\beta}\right)+ \\
& \left(\left|\tilde{e}_{1}\right| \varepsilon^{*}-\left|\tilde{e}_{1}\right| \varepsilon-\frac{1}{\gamma_{2}} \tilde{\varepsilon} \dot{\varepsilon}\right)= \\
& -\frac{1}{2} \hat{\boldsymbol{e}}^{\mathrm{T}} Q_{1} \hat{\boldsymbol{e}}-\frac{1}{2 b} \tilde{\boldsymbol{e}}_{s}^{\mathrm{T}} Q_{2} \tilde{\boldsymbol{e}}_{s}+\sigma \tilde{\beta}\left(\beta-\beta^{0}\right)+\sigma \tilde{\varepsilon}\left(\varepsilon-\varepsilon^{0}\right) .
\end{aligned}
$$

Using $\tilde{\beta}\left(\beta-\beta^{0}\right) \leqslant-\frac{1}{2} \tilde{\beta}^{2}+\frac{1}{2}\left(\beta^{*}-\beta^{0}\right)^{2}$ and $\tilde{\varepsilon}\left(\varepsilon-\varepsilon^{0}\right) \leqslant$ $-\frac{1}{2} \tilde{\varepsilon}^{2}+\frac{1}{2}\left(\varepsilon^{*}-\varepsilon^{0}\right)^{2}$, we have

$$
\begin{aligned}
\dot{V} \leqslant & -\frac{1}{2} \hat{\boldsymbol{e}}^{\mathrm{T}} Q_{1} \hat{\boldsymbol{e}}-\frac{1}{2 b} \tilde{\boldsymbol{e}}_{s}^{\mathrm{T}} Q_{2} \tilde{\boldsymbol{e}}_{s}-\frac{1}{2} \sigma\left(\tilde{\beta}^{2}+\tilde{\varepsilon}^{2}\right)+ \\
& \frac{1}{2} \sigma\left(\left(\beta^{*}-\beta^{0}\right)^{2}+\left(\varepsilon^{*}-\varepsilon^{0}\right)^{2}\right) .
\end{aligned}
$$


Let $\mu=\sigma\left(\left(\beta^{*}-\beta^{0}\right)^{2}+\left(\varepsilon^{*}-\varepsilon^{0}\right)^{2}\right)$. One can guarantee that $\dot{V}$ is negative as long as $\hat{\boldsymbol{e}}$ is outside the compact set $\Omega_{\hat{\boldsymbol{e}}}$ that is defined as

$$
\Omega_{\hat{\boldsymbol{e}}}=\left\{\hat{\boldsymbol{e}}\|\hat{\boldsymbol{e}}\| \leqslant \sqrt{\frac{\mu}{\lambda_{\min }\left(Q_{1}\right)}}\right\} .
$$

According to standard Lyapunov theorem ${ }^{[37]}$, we conclude that $\hat{\boldsymbol{e}}$ is bounded and will converge to $\Omega_{\hat{\boldsymbol{e}}}$. Moreover, the radius of set $\Omega_{\hat{\boldsymbol{e}}}$ can be made arbitrarily small by choosing the smallest eigenvalue of $Q_{1}$ and designing $\beta^{0}$ and $\varepsilon^{0}$. Similarly, signal $\tilde{\boldsymbol{e}}$ is bounded and will converge to $\Omega_{\tilde{\boldsymbol{e}}}$ defined as

$$
\Omega_{\tilde{\boldsymbol{e}}}=\left\{\tilde{\boldsymbol{e}} \mid\|\tilde{\boldsymbol{e}}\| \leqslant \sqrt{\frac{\mu}{\lambda_{\min }\left(Q_{2}\right)}}\right\}
$$

whose radius can be made also arbitrarily small by performing similar operations. The parameter errors $\tilde{\beta}$ and $\tilde{\varepsilon}$ are bounded and will converge to sets $\Omega_{\tilde{\beta}}$ and $\Omega_{\tilde{\varepsilon}}$, respectively. They are defined as

$$
\begin{aligned}
& \Omega_{\tilde{\beta}}=\left\{\tilde{\beta}|| \tilde{\beta} \mid \leqslant \sqrt{\left(\beta^{*}-\beta^{0}\right)^{2}+\left(\varepsilon^{*}-\varepsilon^{0}\right)^{2}}\right\} \\
& \Omega_{\tilde{\varepsilon}}=\left\{\tilde{\varepsilon}|| \tilde{\varepsilon} \mid \leqslant \sqrt{\left(\beta^{*}-\beta^{0}\right)^{2}+\left(\varepsilon^{*}-\varepsilon^{0}\right)^{2}}\right\} .
\end{aligned}
$$

Hence, the radii of sets $\Omega_{\tilde{\beta}}$ and $\Omega_{\tilde{\varepsilon}}$ can be determined by the design parameters $\theta^{0}$ and $\varepsilon^{0}$. By Assumption 2 and $\varepsilon^{*}$ being an unknown constant, the boundedness of $\beta$ and $\varepsilon$ can be guaranteed. From (8), together with (4), (20), and (21), we derive the boundedness of control law $u$.

Remark 4. The parameters update laws (19) and (22) consist of a gradient algorithm along with the $\sigma$ modification term. The addition of this term eliminates the assumption of the persistent excitation and ensures that no parameters drift takes place ${ }^{[28]}$. The design constants $\beta^{0}$ in (19) and $\varepsilon^{0}$ in (22) may be viewed as "the best guesses" of $\beta^{*}$ and $\varepsilon^{*}$, respectively. At the initial stage, the best guesses can make the adaptive parameters fast convergence. Unfortunately, when $\tilde{e}_{1}$ becomes small, the change of $\beta$ (or $\varepsilon$ ) is dominated by the $\sigma$ modification term. This causes $\beta$ (or $\varepsilon$ ) to be driven toward $\beta^{0}$ (or $\varepsilon^{0}$ ). If $\beta^{0}$ (or $\varepsilon^{0}$ ) is not a good guess of the ideal parameter $\beta$ (or $\varepsilon$ ), then the tracking error $e$ may start to increase. To overcome this problem with the $\sigma$ modification, it is possible to modify the update law as $^{[37]}: \sigma=0$, if $\left\|\hat{e}_{1}\right\| \leqslant E_{0} ; \sigma=\sigma_{0}$, otherwise, where $E_{0}$ and $\sigma_{0}$ are design positive constants.

\section{Simulation example}

In this section, numerical simulations are used to illustrate the efficiency of the proposed method. Consider the Duffing forced oscillation system described as

$$
\begin{aligned}
& \dot{x}_{1}=x_{2} \\
& \dot{x}_{2}=-0.1 x_{2}-x_{1}^{3}+12 \cos t+u+d
\end{aligned}
$$

where the external disturbance $d$ is a square wave with amplitude \pm 1 and period $\pi$. Obviously, if $u=0$, this system is chaotic. We now employ our proposed method to design the control law and force the system output $y$ to track the desired reference trajectory $y_{r}=\sin t$. In the phase plane, this reference trajectory is a unit circle $y_{r}^{2}+\dot{y}_{r}{ }^{2}=1$.
Given the positive definite matrices $Q_{1}=\operatorname{diag}\{[1,1]\}$, $Q_{2}=\operatorname{diag}\{[10,10]\}$, the feedback and observer gain vectors are chosen as $K_{c}^{\mathrm{T}}=[2,1]$ and $K_{o}^{\mathrm{T}}=[8,20]$, respectively. By virtue of the SPR-Lyapunov design approach, solving (17) and (18), we can obtain positive definite matrices $P_{1}$ and $P_{2}$. The initial values are chosen as $x_{1}(0)=x_{2}(0)=0.2$, $\hat{x}_{1}(0)=-1.5$, and $\hat{x}_{2}(0)=0.5$. In the control design, the initial parameter values $\beta(0)=10$ and $\varepsilon(0)=8$, the design constants $\gamma_{1}=\gamma_{2}=1$, and $\sigma=0.01$, and the contractionexpansion factors $\alpha_{1}=1-0.97 \exp \left(-e^{2}\right)$ and $\alpha_{2}=1-$ $0.97 \exp \left(-0.8 e^{2}-0.2 \dot{e}^{2}\right)$. The parameters $\beta^{0}$ and $\varepsilon^{0}$ are set to zero. That is, no a priori information about $\beta$ and $\varepsilon$ is available.

For input variables $\hat{e}$ and $\dot{\hat{e}}$ in $\hat{u}_{c}$, we define seven triangular membership functions such as NB, NM, NS, ZE, PS, PM, and PB uniformly distributed on the intervals $[-2,2]$ and $[-8,8]$, respectively, where "N" denotes "negative", "B" denotes "big", "M" denotes "medium", and so on. The used fuzzy rules are listed in Table 1. The simulation results for the proposed control scheme as shown in Figs. 1-4. For a fair comparison, a state-observer based adaptive fuzzy controller is considered, where the ideal control law is approximated by a fuzzy system in the form of $(3)^{[38]}$. For the same control parameters given above, the corresponding simulation results are illustrated in Figs. 5-8.

Table 1 Fuzzy rule base of variable universe adaptive fuzzy control term

\begin{tabular}{cccccccc}
\hline \multirow{2}{*}{$\dot{e}$} & \multicolumn{7}{c}{$\hat{e}$} \\
\cline { 2 - 8 } & NB & NM & NS & ZE & PS & PM & PB \\
\hline NB & -3 & -3 & -3 & -3 & -2 & 0 & 0 \\
NS & -3 & -3 & -3 & -3 & -2 & 0 & 0 \\
NM & -2 & -2 & -2 & -2 & 0 & 1 & 1 \\
ZE & -2 & -2 & -1 & 0 & 1 & 2 & 2 \\
PS & -1 & -1 & 0 & 2 & 2 & 2 & 2 \\
PM & 0 & 0 & 2 & 3 & 3 & 3 & 3 \\
PB & 0 & 0 & 2 & 3 & 3 & 3 & 3 \\
\hline
\end{tabular}

From the simulation results in Figs. 1-4, one can notice that the good tracking performance can be achieved and that the convergence of the system states (position and velocity) to the desired trajectories can be guaranteed in the presence of the unavailable states, function uncertainties, and external disturbances. In addition, the overall signals
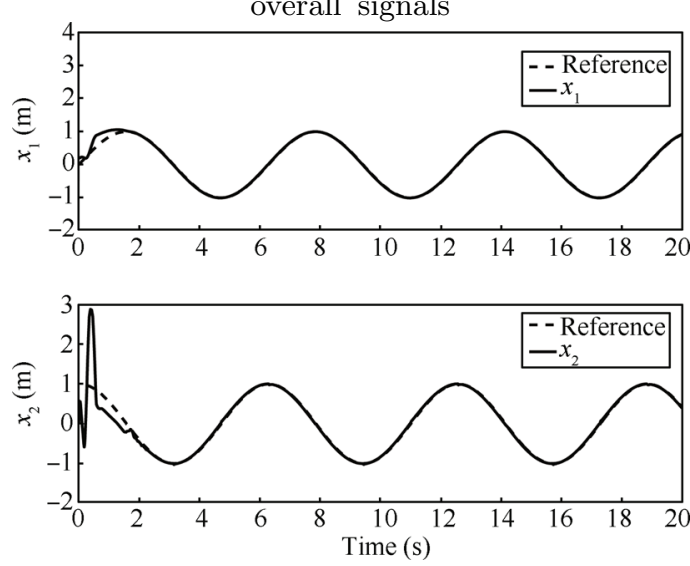

Fig. 1 Tracking curves of states $x_{1}$ and $x_{2}$ using the proposed controller 
in the closed loop system are bounded and the evolution curves of the adaptive parameters are convergent. By using the $\sigma$ modification technique, the parameter drift phenomenon is avoided.
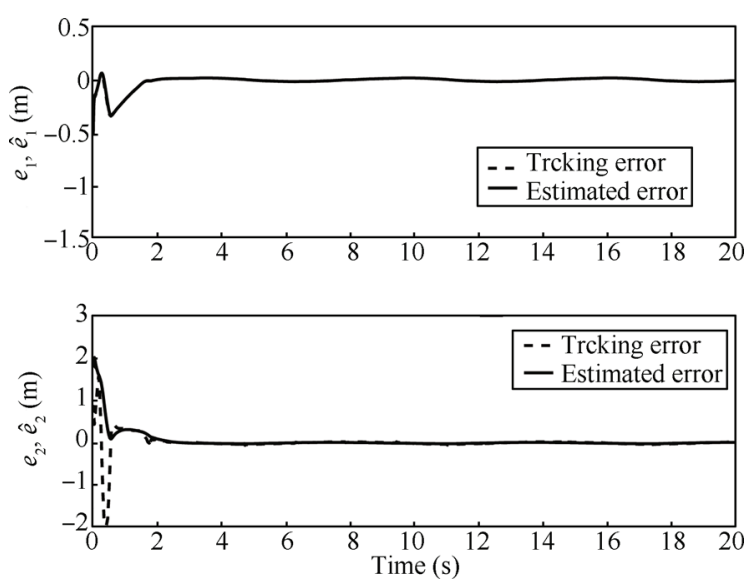

Fig. 2 Tracking error and estimated error curves

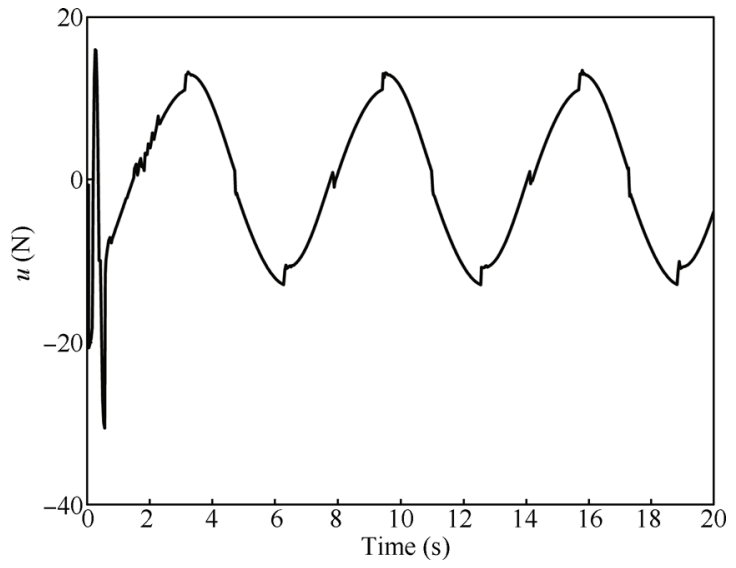

Fig. 3 Control signal curve

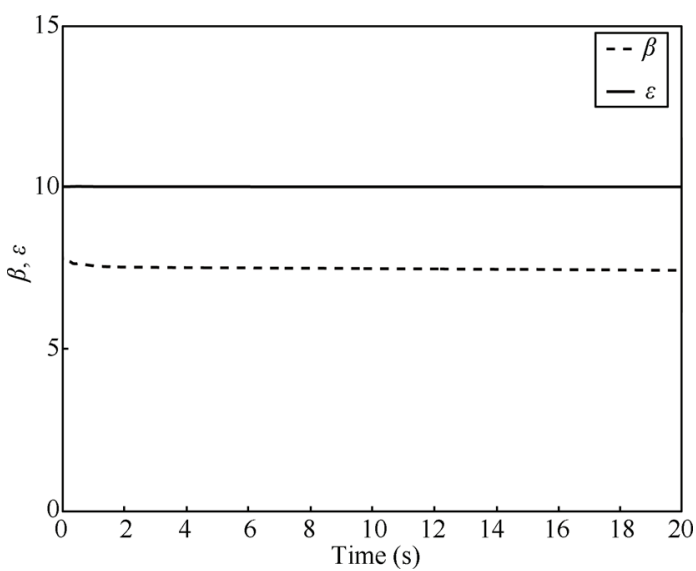

Fig. 4 Evolution curves of the adaptive parameters

Comparing the simulation results, demonstrated in Figs. 5-8, of the state-observed based adaptive fuzzy controller, we notice that the proposed approach yields a smaller tracking error and a faster time response.
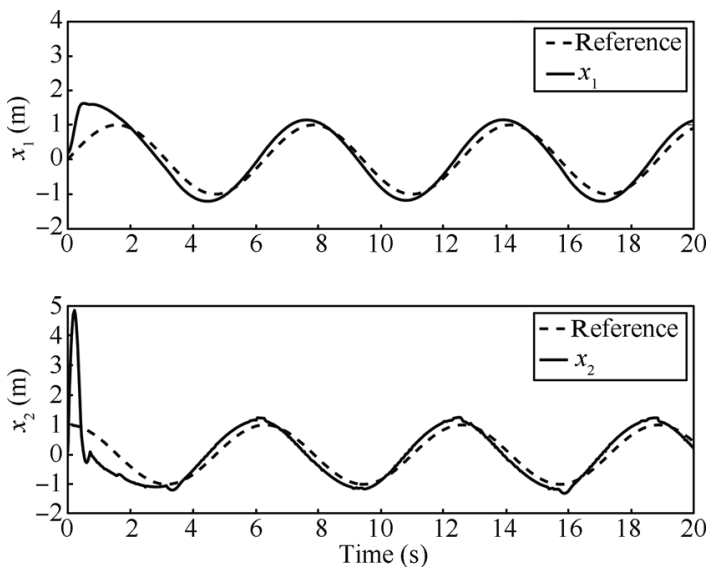

Fig. 5 Tracking curves of states $x_{1}$ and $x_{2}$ using the stateobserved based adaptive fuzzy controller
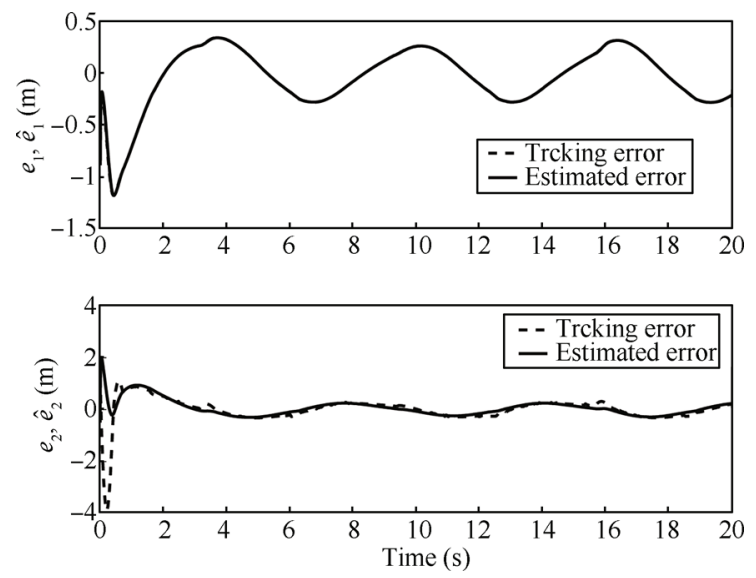

Fig. 6 Tracking error and estimated error curves

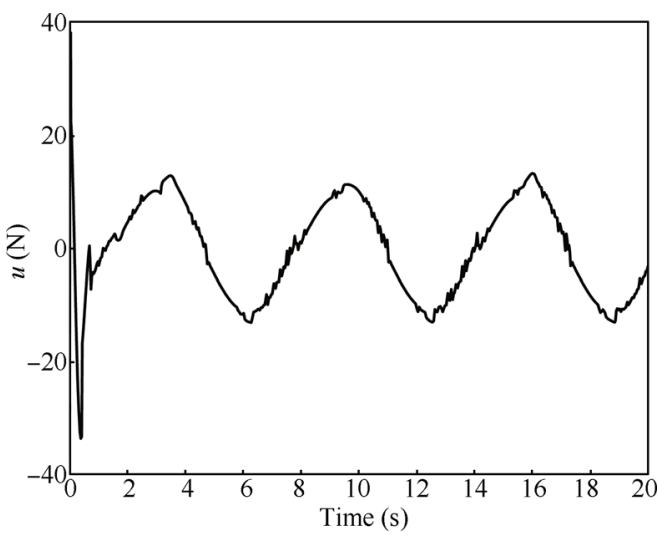

Fig. 7 Control signal curve

\section{Conclusions}

In this paper, an observer-based variable universe adaptive fuzzy control scheme is developed for a class of SISO nonlinear systems with function uncertainties and external disturbances. The scheme, as mentioned in [32,39], belongs to the direct category. The proposed control law consists of a robust control term and a tracking-error observer. Assisted by the tracking-error observer, the state variables do not need to be measurable and a variable universe adaptive fuzzy system is used to approximate the modified ideal 
control law. By virtue of the SPR-Lyapunov design approach, the parameter adaptive laws and the control law are established to stabilize the closed-loop system in the sense that all the involved signals are uniformly ultimately bounded. Simulation results demonstrate the superior performance of the proposed control scheme. The main contributions of this paper are listed as follows: 1) A variable universe adaptive fuzzy control based on the tracking error observer is developed for a class of uncertain nonlinear system with unavailable states. 2) The proposed scheme requires no additional dynamic order since the filtered signals of FBF vector and other control efforts are all included in the lumped uncertainty. The future work may consider nonlinear systems with dead-zone input ${ }^{[40-44]}$.
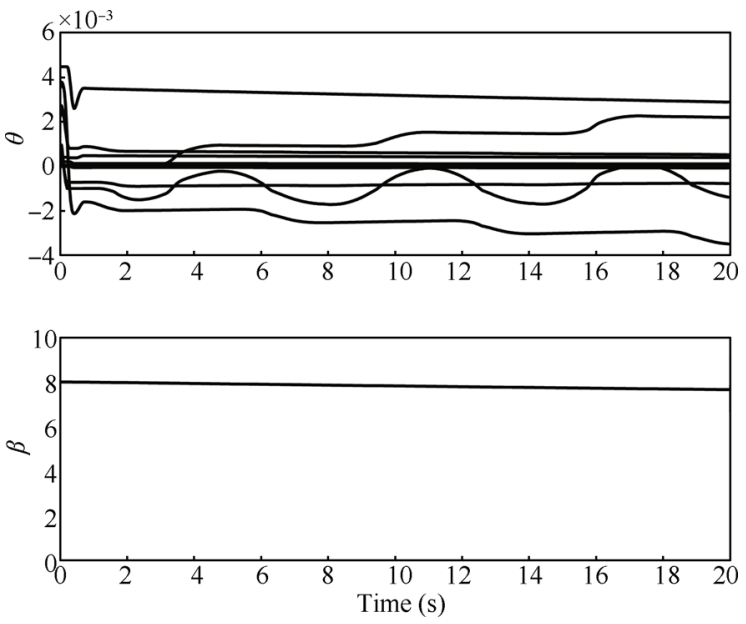

Fig. 8 Evolution curves of the adaptive parameters

\section{References}

[1] L. X. Wang, J. M. Mendel. Fuzzy basis functions, universal approximation, and orthogonal least-squares learning. IEEE Transactions on Neural Networks, vol. 3, no. 5, pp. 807-814, 1992.

[2] A. Boulkroune, M. Tadjine, M. M'Saad, M. Farza. Fuzzy adaptive controller for MIMO nonlinear systems with known and unknown control direction. Fuzzy Sets and Systems, vol. 161, no. 6, pp. 797-820, 2010.

[3] H. F. Ho, Y. K. Wong, A. B. Rad. Robust fuzzy tracking control for robotic manipulators. Simulation Modelling Practice and Theory, vol. 15, no. 7, pp. 801-816, 2007.

[4] Y. C. Chang. Adaptive fuzzy-based tracking control for nonlinear SISO systems via VSS and $H_{\infty}$ approaches. IEEE Transactions on Fuzzy Systems, vol. 9, no.2, pp. 278-292, 2001.

[5] S. Purwar, I. N. Kar, A. N. Jha. Adaptive control of robot manipulators using fuzzy logic systems under actuator constraints. Fuzzy Sets and Systems, vol. 152, no. 3, pp. 651664, 2005.

[6] I. Rojas, H. Pomares, J. Gonzalez, L. J. Herrera, A. Guillen, F. Rojas, O. Valenzuela. Adaptive fuzzy controller: Application to the control of the temperature of a dynamic room in real time. Fuzzy Sets and Systems, vol. 157, no. 16, pp. 2241-2258, 2006.

[7] M. Roopaei, M. Zolghadri Jahromi, R. John, T. C. Lin. Unknown nonlinear chaotic gyros synchronization using adaptive fuzzy sliding mode control with unknown dead-zone input. Communications in Nonlinear Science and Numerical Simulation, vol. 15, no. 9, pp. 2536-2545, 2010.
[8] H. X. Li. Interpolation mechanism of fuzzy control. Science in China Series E-Technological Sciences, vol. 41, no. 3, pp. 312-320, 1998.

[9] A. Sala, T. M. Guerra, R. Babuška. Perspectives of fuzzy systems and control. Fuzzy Sets and Systems, vol. 156, no. 3, pp. 432-444, 2005.

[10] H. T. Yau, J. J. Yan. Adaptive sliding mode control of a high-precision ball-screw-driven stage. Nonlinear Analysis: Real World Applications, vol. 10, no. 3, pp. 1480-1489, 2009.

[11] D. Driankov, H. Hellendoorn, R. Palm. Some research directions in fuzzy control. Theoretical Aspects of Fuzzy Control, John Wiley and Sons, Inc., New York, America, pp. 281312, 1995.

[12] A. Green, J. Z. Sasiadek. Heuristic design of a fuzzy controller for a flexible robot. IEEE Transactions on Control Systems Technology, vol. 14, no. 2, pp. 293-300, 2006.

[13] A. Green, J. Z. Sasiadek. A new optimization method for fuzzy controller's design. Control Theory and Application, vol. 19, no. 2, pp. 279-283, 2002.

[14] Z. K. Zhang, J. Chang. A fuzzy control algorithm with high controlling precision. Fuzzy Sets and Systems, vol. 140, no. 2, pp. 375-385, 2003.

[15] H. X. Li. To see the success of fuzzy logic from mathematical essence of fuzzy control. Fuzzy Systems and Mathematics, vol. 9, no. 4, pp. 1-14, 1995. (in Chinese)

[16] H. X. Li, Z. H. Miao, J. Y. Wang. Variable universe adaptive fuzzy control on the quadruple inverted pendulum. Science in China Series E-Technological Sciences, vol. 45, no. 2, pp. 213-224, 2002.

[17] H. X. Li, J. Y. Wang, Y. B. Feng, Y. D. Gu. Hardware implementation of the quadruple inverted pendulum with single motor. Progress in Natural Science, vol. 14, no. 9, pp. 822-827, 2004.

[18] W. Fang, C. S. Jiang. Adaptive variable universe fuzzy predictive control for aerospace vehicle. Control and Decision, vol. 23, no. 12, pp. 1373-1377, 1388, 2008. (in Chinese)

[19] J. Wang, G. D. Qiao, B. Deng. $H_{\infty}$ variable universe adaptive fuzzy control for chaotic system. Chaos, Solitons and Fractals, vol. 24, no. 4, pp. 1075-1086, 2005.

[20] H. X. Li, Z. H. Miao, E. S. Lee. Variable universe stable adaptive fuzzy control of a nonlinear system. Computers and Mathematics with Applications vol.44, no. 5, pp.799815, 2002.

[21] J. Wang, Z. Zhang, H. Y. Li. Synchronization of FitzHughNagumo systems in EES via $H_{\infty}$ variable universe adaptive fuzzy control. Chaos, Solitons and Fractals, vol. 36, no. 5, pp. 1332-1339, 2008.

[22] Y. F. Wang, C. S. Jiang. Direct adaptive fuzzy sliding mode control with variable universe for near space vehicle. Systems Engineering and Electronics, vol. 33, no. 3, pp. 633637, 2011. (in Chinese).

[23] S. C. Tong, H. X. Li, W. Wang. Observer-based adaptive fuzzy control for SISO nonlinear systems. Fuzzy Sets and Systems, vol. 148, no. 3, pp. 355-376, 2004.

[24] Y. G. Leu, T. T. Lee, W. Y. Wang. Observer-based adaptive fuzzy-neural control for unknown nonlinear dynamical systems. IEEE Transactions on Systems, Man, and Cybernetics. Part B: Cybernetics, vol. 29, no. 5, pp. 583-591, 1999.

[25] Y. G. Leu, W. Y. Wang, T. T. Lee. Observer-based direct adaptive fuzzy-neural control for nonaffine nonlinear systems. IEEE Transactions on Neural Networks, vol. 16 , no. 4, pp. 853-861, 2005.

[26] W. Y. Wang, C. Y. Cheng, Y. G. Leu. An online GA-based output-feedback direct adaptive fuzzy-neural controller for uncertain nonlinear systems. IEEE Transactions on Systems, Man, and Cybernetics, Part B: Cybernetics, vol.34, no. 1, pp. 334-345, 2004. 
[27] J. H. Park, G. T. Park, S. H. Kim, C. J. Moon. Outputfeedback control of uncertain nonlinear systems using a selfstructuring adaptive fuzzy observer. Fuzzy Sets and Systems, vol. 151, no. 1, pp. 21-42, 2005.

[28] A. Boulkroune, M. Tadjine, M. MSaad, M. Farza. How to design a fuzzy adaptive controller based on observers for uncertain affine nonlinear systems. Fuzzy Sets and Systems, vol. 159, no. 8, pp. 926-948, 2008.

[29] A. Boulkroune, M. MSaad. On the design of observer-based fuzzy adaptive controller for nonlinear systems with unknown control gain sign. Fuzzy Sets and Systems, vol. 201, pp. 71-85, 2012.

[30] C. C. Kung, T. H. Chen. Observer-based indirect adaptive fuzzy sliding mode control with state variable filters for unknown nonlinear dynamical systems. Fuzzy Sets and Systems, vol. 155, no. 2, pp. 292-308, 2005.

[31] J. Wang, Q. D. Qiao, B. Deng. Observer-based robust adaptive variable universe fuzzy control for chaotic system. Chaos, Solitons and Fractals, vol. 23, no. 3, pp. 1013-1032, 2005.

[32] H. X. Li. Adaptive fuzzy controllers based on variable universe. Science in China Series E: Technological Sciences, vol. 42, no. 1, pp. 10-20, 1999.

[33] H. G. Guo, H. X. Li, W. J. Zhao, Z. K. Song. Direct adaptive fuzzy sliding mode control with variable universe fuzzy switching term for a class of MIMO nonlinear systems. Mathematical Problems in Engineering, vol. 2012, Article ID 543039, pp. 1-21, 2012.

[34] W. W. Shan, D. M. Jin, Y. Liang. Variable universe adaptive fuzzy logic controller CMOS analog chip implementation. Acta Electronica Sinica, vol. 37, no. 5, pp. 913-917, 2009. (in Chinese)

[35] M. Chemachema. Output feedback direct adaptive neural network control for uncertain SISO nonlinear systems using a fuzzy estimator of the control error. Neural Networks, vol. 36, pp. 25-34, 2012.

[36] J. H. Park, G. T. Park. Adaptive fuzzy observer with minimal dynamic order for uncertain nonlinear systems. IEE Proceedings: Control Theory and Application, vol. 150, no. 2, pp. 189-197, 2003.

[37] P. A. Ioannou, J. Sun. Robust Adaptive Control, PrenticeHall, Englewood Cliffs, NJ, pp. 126-134, 545-576, 1996.

[38] H. G. Guo, H. X. Li, B. J. Zhang. Observer-based direct adaptive fuzzy controller with no additional dynamic order. ICIC Express Letters, vol. 7, no. 12, pp. 3183-3189, 2013.

[39] Y. P. Pan, M. J. Er, D. P. Huang, Q. R. Wang. Adaptive fuzzy control with guaranteed convergence of optimal approximation error. IEEE Transactions on Fuzzy Systems, vol. 19, no. 5, pp. 807-818, 2011.

[40] A. Boulkroune, M. Tadjine, M. MSaad, M. Farza. Adaptive fuzzy observer for uncertain nonlinear systems. Control and Intelligent Systems, vol. 39, no. 3, pp. 145, 2011.

[41] J. Na, X. M. Ren, G. Herrmann, Z. Qiao. Adaptive neural dynamic surface control for servo systems with unknown dead-zone. Control Engineering Practice, vol. 19, no. 11, pp. 1328-1343, 2011.

[42] A. Boulkroune, M. MSaad. A practical projective synchronization approach for uncertain chaotic systems with deadzone input. Communications in Nonlinear Science and $\mathrm{Nu}$ merical Simulation, vol. 16, no. 11, pp. 4487-4500, 2011.

[43] A. Boulkroune, M. MSaad. A fuzzy adaptive variablestructure control scheme for uncertain chaotic MIMO systems with sector nonlinearities and dead-zones. Expert Systems with Applications, vol.38, no. 12, pp. 14744-14750, 2011.

[44] Y. J. Liu, N. Zhou. Observer-based adaptive fuzzy-neural control for a class of uncertain nonlinear systems with unknown dead-zone input. ISA Transactions, vol.49, no. 4 , pp. 462-469, 2010.

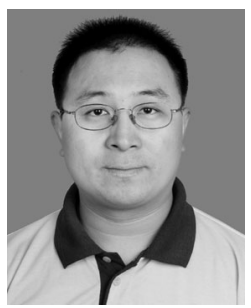

Hai-Gang Guo graduated from Beijing Institute of Machinery, China in 1996. He received the M.Sc. degree from Kunming University of Science and Technology, China in 2004 and the Ph. D. degree from Dalian University of Technology, China in 2013. He is currently a lecturer of School of Mathematics and Statistics, Henan University of Science and Technology, China.

His research interests include fuzzy system and fuzzy control, and uncertainty theory.

E-mail: ghgxsygzy@mail.dlut.edu.cn (Corresponding author)

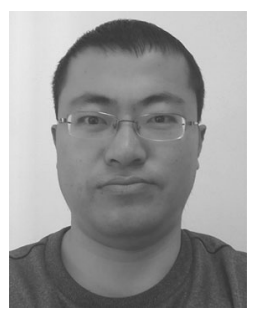

adaptive control.

E-mail: qjupk123@mail.dlut.edu.cn
Bao-Jie Zhang graduated from Binzhou University, China in 1999. He received the M.Sc. degree from Yunnan University of Nationalities, China in 2007. $\mathrm{He}$ is currently a Ph. D. candidate of Dalian University of Technology, China. Moreover, he is a teacher of Qujing Normal University, China.

His research interests include fuzzy control, chaos control and synchronization, and 